\title{
Strategies to Reduce Electronic Health Record Related Burnout Among Nurses
}

\author{
Brian LO ${ }^{\mathrm{a}}$, Jessica KEMP ${ }^{\mathrm{a}, \mathrm{b}}$, Clarel CULLEN ${ }^{\mathrm{a}}$, Tania TAJIRIAN ${ }^{\mathrm{a}, \mathrm{c}}$, \\ Damian JANKOWICZ ${ }^{\mathrm{a}}$ and Gillian STRUDWICK ${ }^{\mathrm{a}, \mathrm{d}, 1}$ \\ anformation Management Group, Centre for \\ Addiction and Mental Health, Toronto, Canada \\ ${ }^{\mathrm{b}}$ Department of Science, University of Waterloo, Waterloo, Canada \\ ${ }^{\mathrm{c}}$ Department of Family and Community Medicine, \\ University of Toronto, Toronto, Canada \\ dinstitute of Health Policy, Management and Evaluation, \\ University of Toronto, Toronto, Canada
}

\begin{abstract}
The introduction of electronic health records (EHR) systems can have a profound impact on the daily clinical activities of nurses. Recent studies have suggested that the adoption of EHR systems may have contributed to burnout among nurses. This poster presentation will explore potential strategies to address the emerging issue of burnout associated with EHR systems in nurses.
\end{abstract}

Keywords. Burnout, electronic health record, nursing informatics, nursing

\section{Introduction}

Nurses are one of the main end-users of health information technologies. Novel technologies, such as patient portals [1] and barcode medication administration systems [2], have influenced how nurses perform their day-to- day clinical activities. In particular, the introduction of electronic health record (EHR) systems in clinical care delivery have led to a number of unintended consequences [3] which may have contributed to burnout. While this issue is well documented among physicians, it is increasingly being recognized among nurses and other health professionals. This poster discusses strategies that organizations can implement to reduce EHR related burnout among nurses.

\section{Methods}

This poster presents information identified in the academic literature regarding strategies to support the potential reduction of EHR related burnout among nurses. Academic databases (e.g., MEDLINE) were searched using relevant key terms (e.g., burnout). Articles that were included in the analysis must focus on strategies that could be applied among nurses to reduce EHR related burnout. Articles that examined EHR burnout in

\footnotetext{
${ }^{1}$ Corresponding Author, Gillian Strudwick, Campbell Family Mental Health Research Institute, Centre for Addiction and Mental Health, Room 7151, 100 Stokes Street, Toronto, Ontario, Canada; E-mail: Gillian.Strudwick@camh.ca.
} 
other healthcare professionals (e.g., physicians, pharmacists) and/or burnout caused by other factors were considered out of scope for this review.

\section{Results}

Before introducing strategies to reduce burnout, a baseline burnout measurement is important to conduct. Strategies to reduce EHR related burnout among nurses include the following two groupings, as well as an evaluation of their efficacy:

\subsection{Understanding EHR Usage Patterns}

Understanding how nurses currently use and complete documentation on EHR systems will provide insight into the issues and bottlenecks related to EHR usage. Gaining a representative and accurate perspective may involve a multifaceted approach that includes delivering validated questionnaires, conducting interviews with point-of- care nurses, and evaluating backend usage data [4]. In particular, backend analytics can provide insights into how nurses are using the different features of the EHR throughout their shift. However, while significant work has focused on identifying meaningful metrics for physicians [5], there is currently limited insight into relevant metrics for nursing practice.

\subsection{Delivery of Evidence-Driven Interventions}

Based on the identified issues and bottlenecks, a number of interventions, such as evidence-driven training and support [6], may be used to support point-of-care nurses in effectively using EHR systems. These interventions may be delivered in collaboration with nurse managers, who have been identified to have a significant role in supporting the effective usage of these technologies [7]. The poster will provide an overview of the various other interventions identified in the literature.

\section{Conclusions}

This poster presentation will present a variety of evidence-informed approaches to addressing the emerging burden associated with using the EHR in clinical care delivery among nurses. As most of the work has focused on physician use of the EHR [8], these findings will provide a unique perspective on the gaps and opportunities to address this problem in nursing practice. Outlining such an approach based on the literature may provide a feasible and data-informed way to iteratively identify and mitigate burnout related to the use of the EHR. A limitation of this work is that the cause of burnout is multifactorial [9], and thus addressing EHR related frustrations and challenges may not eliminate burnout entirely among nurses. By reducing EHR-associated burnout among nurses, it will ensure that EHRs can be appropriately and effectively used to support care delivery. 


\section{Acknowledgements}

The authors would like to acknowledge the Centre for Addiction and Mental Health for their support with this project.

\section{References}

[1] Mayhew C, Strudwick G, Waddell J. Clinical nurse specialists' perceptions of a mental health patient portal. Clin Nurse Spec 32 (2018), 313-322.

[2] Strudwick G, Clark C, McBride B, Sakal M, Kalia K. Thank you for asking: Exploring patient perceptions of barcode medication administration identification practices in inpatient mental health settings. Int $\mathrm{J}$ Med Inform 105 (2017), 31-37.

[3] Koppel R, Wetterneck T, Telles JL, Karsh BT. Workarounds to barcode medication administration systems: their occurrences, causes, and threats to patient safety. J Am Med Inform Assoc 15 (2008), 408423.

[4] Harris DA, Haskell J, Cooper E, Crouse N, Gardner R. Estimating the association between burnout and electronic health record-related stress among advanced practice registered nurses. Appl Nurs Res 43 (2018), 36-41.

[5] Sinsky CA, Rule A, Cohen G et al. Metrics for assessing physician activity using electronic health record log data. J Am Med Inform Assoc 27 (2020), 639-643.

[6] Kadish SS, Mayer EL, Jackman DM et al. Implementation to optimization: A tailored, data-driven approach to improve provider efficiency and confidence in use of the electronic medical record. J Oncol Pract 14 (2018), e421-e428.

[7] Strudwick G, Booth RG, Bjarnadottir RI et al. The role of nurse managers in the adoption of Health Information Technology: Findings from a qualitative study. J Nurs Adm 49 (2019), 549-555.

[8] Collier R. Rethinking EHR interfaces to reduce click fatigue and physician burnout. CMAJ 190 (2018), E994-E995.

[9] MaslachC, Leiter MP. Understanding the burnout experience: recent research and its implications for psychiatry. World Psychiatry 15 (2016), 103-111. 